\section{OC-081 MORTALITY FOLLOWING BLOOD TRANSFUSION FOR NON-VARICEAL UPPER GASTROINTESTINAL BLEEDING}

doi:10.1136/gut.2011.239301.81

ASTaha, ${ }^{1,}{ }^{*}$ CMcCloskey, ${ }^{1}$ TCraigen, ${ }^{1}$ W Angerson, ${ }^{2}$ AA Shah, ${ }^{1}$ C GMorran ${ }^{3}{ }^{1}$ Medicine, Crosshouse Hospital, Kilmarnock, UK; ${ }^{2}$ Surgery, University of Glasgow, Glasgow, UK; ${ }^{3}$ Surgery, Crosshouse Hospital, Kilmarnock, UK

Introduction Blood transfusion is integral to the management of non-variceal upper gastrointestinal bleeding (NV-UGIB), but its safety is being questioned in less severe cases. We, therefore aimed to measure 30-day and 2-year mortality after blood transfusion for NV-UGIB.

Methods This was an observational analysis using routinely collected data at a single hospital in southwest Scotland affiliated to Glasgow University. Cox proportional hazards models were used to estimate the effect of blood transfusion on mortality while adjusting for age, Charlson comorbidity score, the complete Rockall score for acute upper gastrointestinal bleeding, admission status, drug intake, etc. The main outcome measure was death of any cause at 30 days and 2 years after NV-UGIB.

Results 1340 patients presented with NV-UGIB, 808 males (60.3\%), median age 67 years and 564 (42.1\%) were transfused. The overall mortality was $5.3 \%$ at 30 days and $25.8 \%$ at 2 years regardless of transfusion. Mortality was higher in the transfused versus non-transfused patients $(\mathrm{P}<0.001$, log rank test), as shown in figure 1 . Also, in patients with a haemoglobin level greater than $10.0 \mathrm{~g} / \mathrm{dl}$, and comparing subjects who were transfused with those who were not, 30-day mortality (95\% confidence intervals) was $11.5 \%(6.7-18.0)$ versus $3.6 \%$ (2.3-5.3), respectively, $\mathrm{P}<0.001$; and 2 -year mortality $(95 \%$ confidence intervals) was $40 \%(32-49)$ versus $20 \%$ (17-23), P $<0.001$. Adjusted for age, Charlson score, Rockall score and

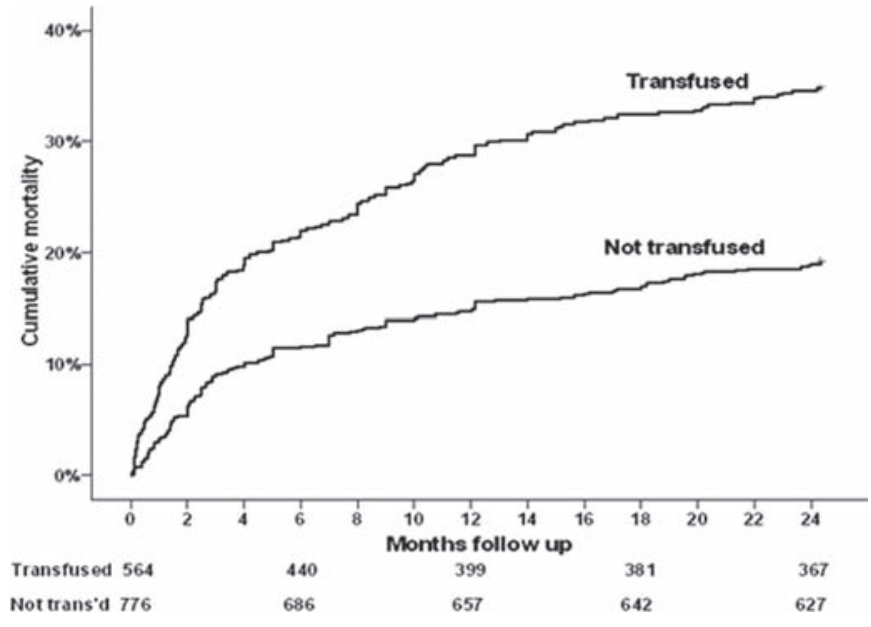

Figure 1 OC-081 The cumulative mortality in transfused versus nontransfused patients $(\mathrm{P}<0.001$, log rank test)

haemoglobin, the hazard ratios (95\% confidence intervals) for death after transfusion was 1.88 (1.00-3.55), $\mathrm{P}=0.051$, at 30 days; and 1.71 (1.28-2.28), $\mathrm{P}<0.001$, at 2 years.

Conclusion In patients with less severe NV-UGIB, mortality is higher following blood transfusion. The need for such intervention should be carefully weighed, and alternative measures should be considered where appropriate, particularly in less severe cases.

Competing interests None.

Keywords gastrointestinal bleeding, mortality, outcomes. 